\title{
A Pharmacokinetic/Pharmacodynamic Model to Predict Effective HIV Prophylaxis Dosing Strategies for People Who Inject Drugs $\mathbf{s}$
}

\author{
Katy L. Garrett, ${ }^{1}$ Jingxian Chen, ${ }^{2}$ Brian M. Maas, ${ }^{2}$ Mackenzie L. Cottrell, Heather A. Prince, \\ Craig Sykes, Amanda P. Schauer, Nicole White, and Julie B. Dumond \\ Division of Pharmacotherapy and Experimental Therapeutics, UNC Eshelman School of Pharmacy (K.L.G., J.C., B.M.M., \\ M.L.C., C.S., A.P.S., J.B.D.), and School of Medicine (H.A.P., N.W.), University of North Carolina at Chapel Hill, Chapel Hill, \\ North Carolina
}

Received May 30, 2018; accepted August 22, 2018

\begin{abstract}
The goal of this work was to evaluate dosing strategies for tenofovir disoproxil fumarate (TDF), tenofovir alafenamide (TAF), and emtricitabine (FTC) for pre-exposure prophylaxis (PrEP) with injection drug use with a pharmacokinetic/pharmacodynamics analysis of concentration data generated from two single-dose clinical studies conducted in healthy women. Population pharmacokinetic models were developed using measured intracellular metabolite, endogenous nucleotide competitors, and extracellular parent drug concentrations. Intracellular metabolite concentrations were normalized to endogenous competitors and compared with an $\mathrm{EC}_{90}$ target for PrEP efficacy. Monte Carlo simulations were used to select effective dose strategies of single agents (TAF, TDF, and FTC) and combinations (TDF + FTC and TAF + FTC).
\end{abstract}

Daily, intermittent, and event-driven dosing regimens at varying dosage amounts were explored. When combined, TDF + FTC and TAF + FTC both provided quick ( 0.5 hours) and durable (up to 84 and 108 hours, respectively) protection of $\geq 99 \%$ after a single dose. When dosed twice per week, protection remained at $100 \%$. Single-agent regimens provided lower estimates of protection than either combination tested. Here, the application of pharmacokinetic modeling to in vitro target concentrations demonstrates the added utility of including FTC in a successful PrEP regimen. While no TAF-based PrEP data are currently available for comparison, this analysis suggests TAF + FTC could completely protect against percutaneous exposure with as little as two doses per week.

\section{Introduction}

As part of a comprehensive harm reduction program for people who inject drugs [(PWID), opioid treatment facilities, syringe services programs, etc.], the Centers for Disease Control and Prevention recommends pre-exposure prophylaxis (PrEP) with once daily tenofovir disoproxil fumarate (TDF), alone or in combination with emtricitabine (FTC), to prevent human immunodeficiency virus (HIV) (US Public Health Service, 2014). This recommendation is based on the Bangkok Tenofovir Study (Choopanya et al., 2013), which

This work was supported by the National Institutes of Health National Institute of Allergy and Infectious Disease [Grant U01 AI095031]; the Centers for AIDS Research [Grant P30 AI050410]; the National Institutes of Health National Institute of General Medical Sciences [Grant T32 GM086330]; Gilead Sciences, Inc. [Grant IN-US-120-D001]; and the National Institutes of Health National Center for Advancing Translational Sciences [Grant UL1TR001111].

Portions of this work were presented at HIV Research for Prevention (HIVR4P) [Oct 17-21, 2016, Chicago, IL, Poster P21.12LB] and American Conference on Pharmacometrics 8 [Oct 14-19, 2017, Fort Lauderdale, FL, Poster T-081]

${ }^{1}$ Current affiliation: Maine Medical Center, Portland, Maine.

${ }^{2}$ Current affiliation: Merck \& Co., Inc., Kenilworth, New Jersey.

https://doi.org/10.1124/jpet.118.251009.

S This article has supplemental material available at jpet.aspetjournals.org. found a $49 \%$ risk reduction in participants taking TDF compared with placebo. The combination of TDF + FTC has never been studied in a PWID population, despite showing benefit in other settings (Grant et al., 2010; Baeten et al., 2012).

TDF and FTC are known to work synergistically (Cottrell et al., 2016), have different pharmacokinetic (PK) profiles in various tissues (Patterson et al., 2011; Cottrell et al., 2015; Dickinson et al., 2015), and are available in a coformulated product. Additionally, the combination is attractive in the event of exposure to HIV with resistance-associated mutations. Tenofovir alafenamide (TAF) was approved as a coformulated tablet with FTC in 2016. When compared with TDF, TAF has a more advantageous PK and safety profile (Ruane et al., 2013) in the treatment of HIV, and is currently in clinical trials for its use in preventing HIV transmission from sexual exposure. Given these facts, the feasibility of TAF + FTC for prevention of HIV transmission for PWID is appealing; however, the efficacy of TAF for PrEP is still unknown.

The Bangkok Tenofovir Study was largely conducted in a directly observed therapy setting. While directly observed

ABBREVIATIONS: BQL, below the quantitation limit; dATP, deoxyadenosine triphosphate; dCTP, deoxycytidine triphosphate; FTC, emtricitabine; FTCtp, emtricitabine triphosphate; HIV, human immunodeficiency virus; IIV, interindividual variability; PBMC, peripheral blood mononuclear cell; PD, pharmacodynamic; PK, pharmacokinetic; PrEP, pre-exposure prophylaxis; PWID, people who inject drugs; TAF, tenofovir alafenamide; TDF, tenofovir disoproxil fumarate; TFV, tenofovir; TFVdp, tenofovir diphosphate. 
therapy can improve HIV virologic outcomes in PWID (Altice et al., 2007; Berg et al., 2011), it may not be a sustainable option for PrEP outside of a medication-assisted treatment program for opioid dependence. Antiretroviral therapy and engagement in care, for HIV treatment or prevention, can be especially difficult for people with substance use disorder, with or without other mental health disorders. The use of event-driven PrEP among the men who have sex with men population has shown benefit (Molina et al., 2015), but on-demand dosing has not yet been studied in PWID.

TAF, TDF, and FTC are metabolized to active intracellular metabolites by host phosphatases (Anderson et al., 2011). Tenofovir diphosphate (TFVdp) is the active metabolite of both TAF and TDF, and FTC triphosphate (FTCtp) is the metabolite of FTC. These metabolites work by acting as nucleotide analogs and are reverse transcribed into the HIV DNA in HIV-infected cells. Incorporation of either TFVdp or FTCtp into HIV DNA results in chain termination and prevention of viral replication. To be incorporated into HIV DNA, these metabolites must compete with their analogous, naturally occurring nucleotide, referred to here as endogenous nucleotides. TFVdp is an adenosine triphosphate analog, and FTCtp is a cytosine triphosphate analog. Both metabolites have long intracellular half-lives: about 6 days for TFVdp and 1.6 days for FTCtp (Anderson et al., 2011). Since PWID would be exposed to HIV via direct inoculation in blood (rather than through tissues as in sexual exposure), drug concentrations at that site, i.e., peripheral blood mononuclear cells (PBMCs) were considered especially relevant. To investigate the potential benefit of TDF or TAF, with or without FTC, PK modeling of the active metabolites and their endogenous nucleotide competitors, deoxyadenosine triphosphate (dATP) and deoxycytidine triphosphate (dCTP), in PBMCs was used in combination with a previously described pharmacodynamic (PD) model (Cottrell et al., 2016) to simulate effective dosing regimens in a PWID population.

\section{Materials and Methods}

Study Design. Concentrations of tenofovir (TFV), TAF, FTC, TFVdp, and FTCtp from two previously reported oral, single-dose PK studies in healthy women (NCT010330199 and NCT02357602) (Cottrell et al., 2016, 2017) were analyzed. Both studies were approved by the University of North Carolina's Biomedical Institutional Review Board. Details of the study design and bioanalytical analysis were described previously (Cottrell et al., 2016, 2017). Briefly, 72 women were enrolled to receive either TDF (150, 300, or $600 \mathrm{mg} ; n=8 / \mathrm{arm})$, FTC (100, 200, $400 \mathrm{mg} ; n=8 / \mathrm{arm})$, or TAF (5, 10, $25 \mathrm{mg} ; n=8 / \mathrm{arm})$. For TDF and FTC, plasma was collected at $0.5,1,2,3,4,6,9,12,18,24$, 36 , and 48 hours postdose; PBMCs were collected at 1, 3, 6, 12, 24, 36, and 48 hours postdose. For TAF, plasma was collected 1, 3, 6, 12, 24, $72,168,240$, and 336 hours postdose and PBMCs were collected 3, 6, $12,24,72,168,240$, and 336 hours postdose. All analytes were measured by liquid chromatography-tandem mass spectrometry methods with the following lower limits of quantitation: TFV and FTC (5 ng/ml); TFVdp, FTCtp, dATP, and dCTP $(0.02 \mathrm{ng} / \mathrm{ml}$, normalized by cell count); and TAF $(0.05 \mathrm{ng} / \mathrm{ml})$. TAF was only measured in subjects enrolled in the $25 \mathrm{mg}$ arm.

Doses of TDF, FTC, and TAF and concentrations were converted to micromolar units to allow comodeling of all analytes. The molecular weights used for conversion were: TDF, $635.52 \mathrm{~g} / \mathrm{mol}$; FTC, $247.248 \mathrm{~g} / \mathrm{mol}$; TAF, $476.466 \mathrm{~g} / \mathrm{mol}$; and TFV, $287.216 \mathrm{~g} / \mathrm{mol}$. The estimate of
PBMC volume was one cell equivalent to $282 \mathrm{fl}$ (Rodriguez et al., 2000).

Model Development and Evaluation. Parent (TFV, TAF, and FTC) and metabolite (TFVdp and FTCtp) data were comodeled in NONMEM (version 7.3; ICON Development Solutions, Ellicott City, $\mathrm{MD})$ using the ADVAN6 subroutine and the first-order conditional (TDF and FTC) or Laplacian (TAF) estimation method with interaction. Pirana and Perl-speaks-NONMEM were used for the population model development (Lindbom et al., 2005; Keizer et al., 2011). Data visualization was conducted in $\mathrm{R}$ (version 3.3.2; $\mathrm{R}$ Foundation for Statistical Computing, Vienna, Austria) using R Studio (version 1.0.136; RStudio, Boston, MA) (R Core Team, 2016) and the tidyverse and Xpose 4 packages.

Models tested included one-, two-, and three-compartment plasma models with zero- and first-order absorption and elimination. Conversion of parent to metabolite was tested using firstorder and Michaelis-Menten kinetics (Chen et al., 2016) and transit compartments. Parent amounts, assumed to be primarily renally excreted rather than metabolized, were linked to metabolite concentrations using microrate constants. The M3 method was used to account for data below the quantitation limit (BQL) (Beal, 2001).

Interindividual variability (IIV) was assumed to be normally distributed with a mean of zero and variance $\omega^{2}$, and exponentially associated with the population parameters. Covariance among IIVs was explored based on correlation. Proportional and combined additive and proportional residual variability models were assessed separately for each analyte, and were assumed to be normally distributed with a mean of zero and a variance $\sigma^{2}$. Due to the homogeneity of the study population, covariate analysis was not performed. Final model selection was determined using a combination of the precision of parameter estimates, Akaike information criterion, goodness-of-fit plots, and physiologic plausibility. Prediction-corrected visual predictive checks were generated using nominal sample times to evaluate model appropriateness.

Simulations. Monte Carlo simulations of TFVdp and FTCtp in PBMCs were performed for 1000 virtual subjects, each taking $300 \mathrm{mg}$ TDF, $200 \mathrm{mg}$ FTC, or $25 \mathrm{mg}$ TAF (conventional treatment doses for each drug) based on the final model developed for each drug. Dosing regimens included conventional treatment dose, double dose (i.e., a dose of $600 \mathrm{mg}$ TDF, $400 \mathrm{mg}$ FTC, or $50 \mathrm{mg}$ TAF), steady-state dosing with one to seven equally spaced conventional treatment doses/week, and on-demand dosing in which subjects took a double dose 24 or 2 hours prior to exposure followed by a single, standard treatment dose 24 and 48 hours after exposure (Molina et al., 2015). dCTP and dATP concentrations were randomly selected from the log-normal distribution of those analytes, presented as log[analyte] $N$ (mean, S.D.); $\log [\mathrm{dCTP}] \sim N(0.35,0.47)$; and $\log [\mathrm{dATP}] \sim N(-0.99,0.38)$. Ratios of TFVdp to dATP and FTCtp to dCTP were subsequently calculated by dividing the concentration of metabolite by the concentration of endogenous nucleotide.

Protective Effect. The active metabolite to endogenous nucleotide ratio that was $90 \%$ effective $\left(\mathrm{EC}_{90}\right)$ in prevention of transmission ( 0.29 for TFVdp:dATP and 0.07 for FTCtp:dCTP) was used when any of the drugs were assessed as monotherapy (Cottrell et al., 2016). A PD interaction model (eq. 1) of TFVdp:dATP and FTCtp: dCTP ratios was used to determine the simulated protected effect when each of the TFV prodrugs was combined with FTC. The 50\% effective concentration $\left(\mathrm{EC}_{50}\right)$ and Hill $(\mathrm{H})$ coefficients for each drug ratio and the synergy parameter $(\psi)$ were previously established and fixed (Cottrell et al., 2016). The parameters were: $\mathrm{EC}_{50}$, TFVdp $=0.086$, $\mathrm{H}_{\text {TFVdp }}=1.81 ; \mathrm{EC}_{50}$, FTCtp $=0.077, \mathrm{H}_{\text {FTCtp }}=1.88$; and $\psi=0.63$. Because the active metabolites have long half-lives, to allow for consistency in modeling and to align with clinical standards for trough monitoring (i.e., vancomycin), ratios were assessed 30 minutes prior to the next dose for steady-state dosing. If ratios were greater than the $\mathrm{EC}_{90}$ value, they were declared above target for the dosing interval 


$$
E=\frac{\left[\mathrm{TFV} /\left(\psi \times \mathrm{EC}_{50, \mathrm{TFVdp}}\right)\right]^{\mathrm{H}_{\mathrm{TFVdp}}}+\left[\mathrm{FTC} /\left(\psi \times \mathrm{EC}_{50, \mathrm{FTCtp}}\right)\right]^{\mathrm{H}_{\mathrm{FTCtp}}}+\left[\mathrm{TFV} /\left(\psi \times \mathrm{EC}_{50, \mathrm{TFV} d \mathrm{p}}\right)\right]^{\mathrm{H}_{\mathrm{TFVdp}}} \times\left[\mathrm{FTC} /\left(\psi \times \mathrm{EC}_{50, \mathrm{FTCtp}}\right)\right]^{\mathrm{H}_{\mathrm{FTCtp}}}}{1+\left[\mathrm{TFV} /\left(\psi \times \mathrm{EC}_{50, \mathrm{TFVdp}}\right)\right]^{\mathrm{H}_{\mathrm{TFVdp}}}+\left[\mathrm{FTC} /\left(\psi \times \mathrm{EC}_{50, \mathrm{FTCtp}}\right)\right]^{\mathrm{H}_{\mathrm{FTCtp}}}+\left[\mathrm{TFV} /\left(\psi \times \mathrm{EC}_{50, \mathrm{TFV} d \mathrm{p}}\right)\right]^{\mathrm{H}_{\mathrm{TFV} d p}} \times\left[\mathrm{FTC} /\left(\psi \times \mathrm{EC}{ }_{50, \mathrm{FTCtp}}\right)\right]^{\mathrm{H}_{\mathrm{FTCtp}}}}
$$

\section{Results}

Study Population and PK Observations. Of the 288 TFV plasma samples collected, one $(0.3 \%)$ was BQL. One participant in the FTC arm had unusable samples due to improper storage, leaving 276 available for analysis and one (0.4\%) was BQL. There were 166 and 161 TFVdp and FTCtp samples for analysis in the TDF and FTC arms, respectively. Five (3.6\%) TFVdp samples and one (0.6\%) FTCtp sample were BQL. TAF was measured in plasma from participants taking $25 \mathrm{mg}$ TAF. All samples collected beyond 6 hours postdose were BQL, resulting in 23 samples for evaluation. Of these, five $(22 \%)$ were BQL, all occurring at the 6-hour time point. Women enrolled in both studies had median ages of 22 and 27 years (Table 1). There were no significant differences in age or weight between the groups $(P=0.1)$. The majority of participants were white $(72 \%)$ and non-Hispanic (96\%).

Population PK Analysis. A two-compartment model with first-order absorption and elimination best described the FTC plasma pharmacokinetics, with saturable metabolite formation. A one-compartment model described FTCtp, with first-order transfer of metabolite back to plasma. A twocompartment model with delayed absorption best described the plasma TFV (from TDF) pharmacokinetics. A onecompartment model with first-order formation from plasma TFV and clearance out of the body best described the TFVdp data. The elimination rate of TFVdp following dosing with TDF could not be estimated because its half-life was longer than the sampling period of 48 hours, so it was fixed to a value consistent with the TAF data $\left(0.0125\right.$ hour $\left.^{-1}\right)$. A onecompartment model with an additional transit compartment for metabolite conversion best described the TAF and TFVdp data. Since the time to maximum concentration is 0.48 hours after dosing (Ruane et al., 2013) and the earliest time point in this study was 1 hour, the absorption phase could not be characterized and the rate constant was fixed to $2.8 \mathrm{hour}^{-1}$.

Simulated concentration-time profiles for TFV and TFVdp (TDF), FTC and FTCtp (FTC), and TAF and TFVdp (TAF) following dosing with TDF $300 \mathrm{mg}$, FTC $200 \mathrm{mg}$, and TAF $25 \mathrm{mg}$ are shown in Fig. 1. Population PK parameter estimates are provided in Supplemental Table 1, model schematics for each drug are presented in Supplemental Figs. 1-3, and an example model code is presented in Supplemental File 1. All fixed effect parameters were estimated with acceptable precision (percentage of residual S.E. $\leq 30 \%$ ). Shrinkage of random effects parameters was $\leq 50 \%$. Diagnostic plots did not reveal any model misspecification and estimated parameters were physiologically plausible. The IIV for the clearance parameter was higher in patients receiving TAF $(>65 \%)$ compared with those receiving TDF or FTC $(<35 \%)$. All three models captured the central tendency of the data well and were deemed suitable to generate simulations.

Simulations and Efficacy. With on-demand dosing, a single dose of TDF is predicted to provide protection in $2 \%$ and $50 \%$ of the population at the time of HIV exposure if TDF is taken 2 or 24 hours prior (Fig. 2). All other dosing combinations are expected to provide near $100 \%$ protection at time of exposure, regardless of initial dose time. The TDF + FTC dose provides protection for up to 120 hours postexposure, whereas TAF + FTC provides protection for 192 hours postexposure in $>99 \%$ of the population. At steady state, the model predicts that TDF alone provides the least efficacy based on the target $\mathrm{EC}_{90}$ (Fig. 3). Taking one, four, or seven doses per week of TDF is predicted to provide $3 \%, 72 \%$, and $92 \%$ protection, respectively. Taking the same number of TAF doses per week is predicted to provide $16 \%, 100 \%$, and $100 \%$ protection, respectively. FTC is predicted to provide similar efficacy as TAF. When combining FTC with either TDF or TAF, as few as two doses per week should provide near $100 \%$ protection.

A single dose of TDF is expected to maximally provide $44 \%$ protection at 48 hours after dosing. (Fig. 4) Comparatively, a single dose of TAF would provide $100 \%$ protection between 3 and 36 hours after the dose with $>90 \%$ protection 60 hours after the dose. FTC would provide $>90 \%$ protection between 0.5 and 84 hours after a dose. Using twice the clinical dose, the time to maximal protection with TDF is shortened to 28 hours, where $80 \%$ protection is expected. TAF should provide near $100 \%$ protection 2 hours after the dose and remain near $100 \%$ through 72 hours, with $90 \%$ protection through

TABLE 1

Demographics of the participants

\begin{tabular}{lccc}
\hline Demographics of the Participants & TDF $(n=24)$ & FTC $(n=24)$ & TAF $(n=24)$ \\
\hline Age, $\mathrm{yr}^{a}$ & $27(21-38)$ & $22(20-39)$ & $25(19-46)$ \\
Weight, $\mathrm{kg}^{a}$ & $66.85(50.8-94.7)$ & $62.75(46.3-90.3)$ & $68.54(50.53-107.05)$ \\
Race & & & \\
White & $16(66.7)$ & $18(75)$ & $20(83.3)$ \\
Black & $7(29.2)$ & $4(16.7)$ & $4(16.7)$ \\
Asian & $1(4.2)$ & $1(4.2)$ & 0 \\
Native American & 0 & $1(4.2)$ & 0 \\
Ethnicity & & & $3(12.5)$ \\
Hispanic & 0 & 0 & $21(87.5)$ \\
Non-Hispanic & 24 & 24 & \\
\hline
\end{tabular}

${ }^{a}$ Data expressed as median (minimum-maximum).

${ }^{b}$ Data expressed as number (percentage). 

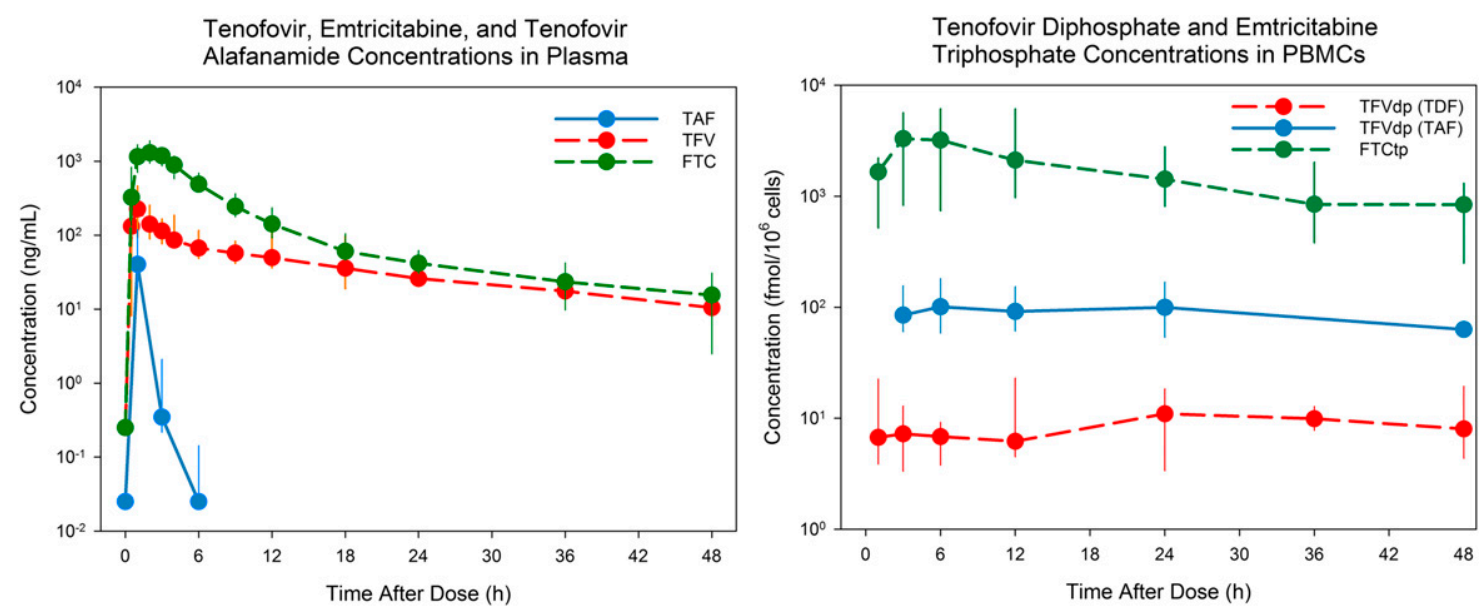

Fig. 1. Concentrations of parent drug and metabolites in plasma and PBMCs. Median (minimum-maximum) concentration of TFV, FTC, TAF, TFVdp, and FTCtp in plasma (left) and PBMCs (right) after a single dose of $300 \mathrm{mg}$ TDF, $200 \mathrm{mg}$ FTC, or $25 \mathrm{mg}$ TAF. All data are represented for TDF and FTC; TAF data are represented through 48 hours of study (total study, 14 days; 48-hour data point imputed).

120 hours. FTC provides near $100 \%$ protection through 144 hours. Combining FTC with TDF or TAF results in protection $>90 \%$ for 144 and 176 hours after dosing, respectively.

\section{Discussion}

TFVdp and FTCtp work by acting as nucleotide analogs and are reverse transcribed into the HIV DNA. Incorporation of either TFVdp or FTCtp results in HIV DNA chain termination and inhibition of viral replication. TFVdp and FTCtp compete with their endogenous nucleotides dATP and dCTP. When TFVdp and FTCtp are present, they must reach a threshold to be preferentially incorporated into DNA. Because this threshold is inherently dependent upon the endogenous nucleotide pool within an individual, we chose to use the ratio of TFVdp to dATP and FTCtp to dCTP as our PD targets. This method has been previously validated using in vitro data (Cottrell et al., 2016) and was cited as an efficacy target in a nonhuman primate PrEP study (García-Lerma et al., 2011).

In this analysis, we show that the rapid accumulation of FTCtp and the prolonged half-life of TFVdp in PBMCs are both necessary to protect PWID from HIV infection. Complete protection could be achieved with either TDF + FTC or TAF + FTC dosed twice weekly for those engaged in frequent and routine injection drug use, or dosed on-demand as little as 2 hours prior to exposure for those who inject less frequently. Our data support the Centers for Disease Control and Prevention recommendation of TDF alone as a viable option, where our prediction of daily TDF would provide $92 \%$ protection. For patients unable to take TDF, daily TAF could provide complete protection. However, when TDF or TAF are combined with FTC, additional individuals will be protected if using fewer doses. Due to the synergistic efficacy of FTCtp and TFVdp (Cottrell et al., 2016), a combination product could protect $100 \%$ of the population with two doses per week with a single dose providing protection at least 84 hours after a dose.

This analysis was based on the assumption of preventing infections caused by blood-borne transmission of wild-type HIV. Nucleoside reverse transcriptase inhibitor associated mutations, such as M184V and K65R, generally decrease viral fitness (Wertheim et al., 2017). However, the M184V mutation, which confers FTC resistance but increases TDF/TAF sensitivity, is common among people failing antiretroviral
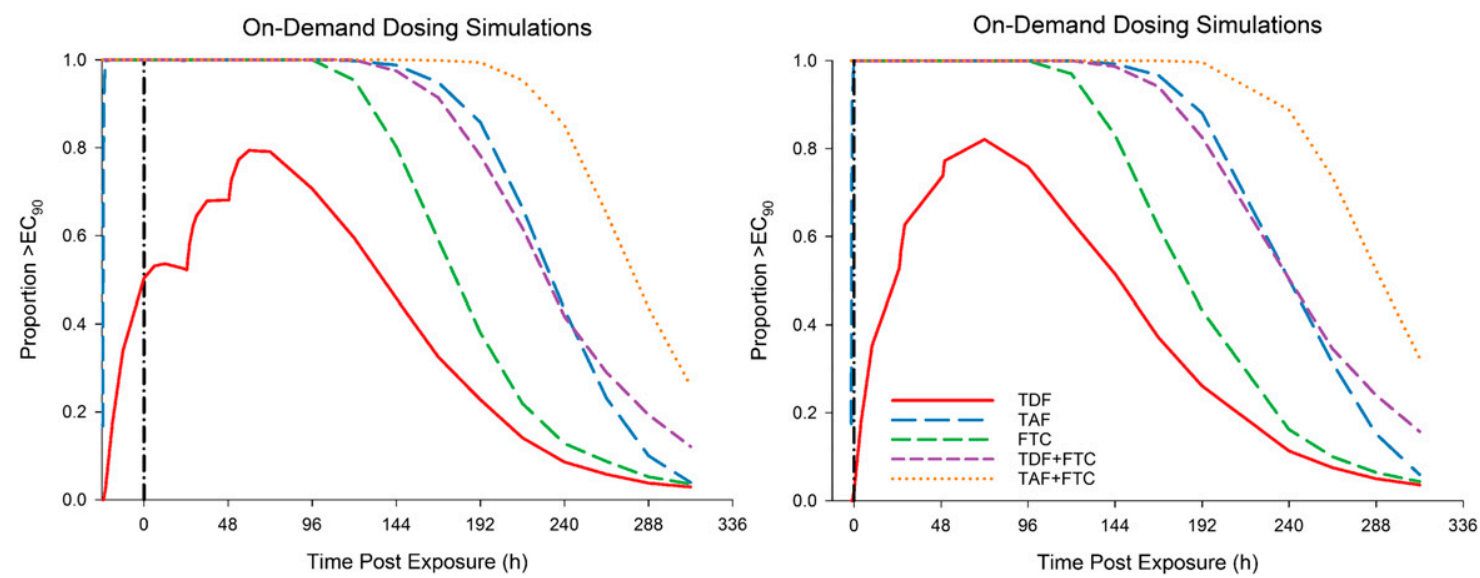

Fig. 2. Protection using on-demand dosing. Depicted are PK/PD simulations of protection using on-demand dosing in which a double dose of the indicated drug is taken 24 hours (left) or 2 hours (right) prior to HIV exposure followed by standard doses 24 and 48 hours after exposure. Standard doses: $300 \mathrm{mg}$ TDF, $25 \mathrm{mg}$ TAF, and $200 \mathrm{mg}$ FTC. 


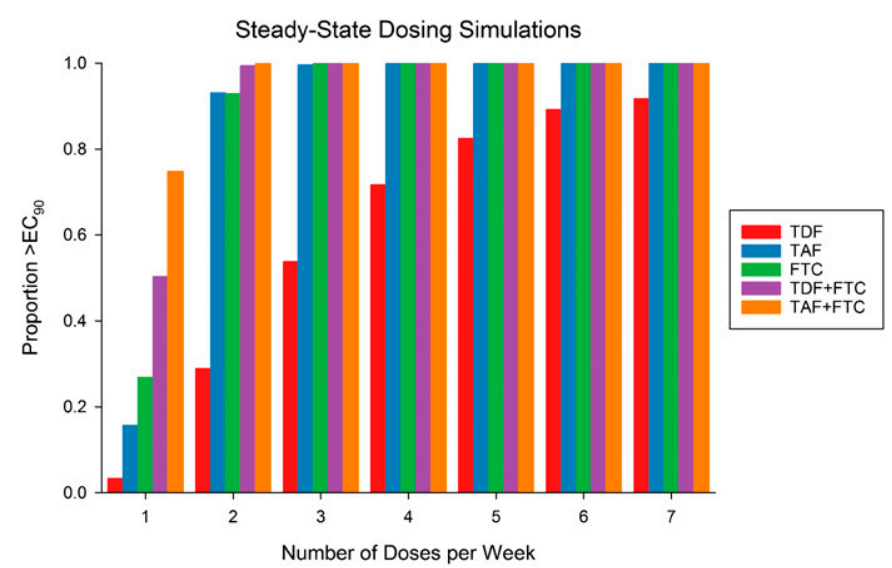

Fig. 3. Protection using steady-state dosing. Depicted is the proportion of population above the target at the end of the dosing interval in 1-7 equally spaced doses of the target drug each week.

therapy (Wainberg et al., 2011). Primary resistance with M184V can occur in $7 \%-23 \%$ of new HIV infections (Wainberg et al., 2011), thus PrEP with FTC alone is not recommended in practice. Despite perfect adherence, PrEP may not be able to protect against multidrug resistant virus (Knox et al., 2017).

We chose to evaluate protection conferred by a single conventional treatment dose of each drug and combination. A $300 \mathrm{mg}$ dose of TDF was expected to provide a maximum of $44 \%$ protection, whereas FTC, TAF, TDF + FTC, and TAF + FTC all reached $100 \%$ protection and sustained it for up to 3.5 days after dosing. To determine if weekly dosing was a viable option, double doses of all drugs and combinations were tested. At 7 days after a double dose of TAF + FTC, 93\% of the population is expected to be protected compared with $24 \%$, $38 \%, 53 \%$, and $78 \%$ with TDF, FTC, TAF, and TDF + FTC, respectively.

Additionally, we chose to look at multidose and event-driven strategies for each drug and combination: evaluating one to seven doses per week and utilizing an event-driven strategy around the time of injection. On-demand strategies were evaluated starting 2 or 24 hours prior to injection. FTC, TAF, TDF + FTC, and TAF + FTC were estimated to provide
$100 \%$ protection whether taken 2 or 24 hours prior to event with protection up to 192 hours for TAF + FTC. Conversely, due to the much lower TFVdp concentrations associated with TDF than TAF, TDF was estimated to only protect $2 \%$ and $50 \%$ if taken 2 or 24 hours prior to event, respectively. With three doses per week TAF and FTC were estimated to provide $100 \%$ protection, whereas the same level of protection could be achieved with two doses per week of TAF + FTC or TDF + FTC.

Pharmacometric models are limited by the data used to generate them. For the TDF model, a 48-hour sampling window was not long enough to capture the elimination phase of TFVdp due to its extended half-life. We fixed this parameter to a value comparable to the one from the TAF model. In addition, the first-order process failed to capture the rapid generation of TFVdp within the first 2 hours, while using the saturable model only described it well at the $300 \mathrm{mg}$ dose level. We chose the model with first-order TFVdp conversion given that it fit the data across doses better and is easier to interpret. The model is able to reasonably predict the TFVdp steady-state trough concentrations (Chen et al., 2016), which were more relevant for this exercise. FTC had minimal limitations since sampling times allowed for adequate estimation of absorption and elimination of both FTC and FTCtp. Additionally, nonlinearity was observed in the dose range studied and implemented in the model.

For TAF model development, only the highest dosing arm (25 mg) had detectable TAF plasma concentrations and the earliest time point was 1 -hour postdose. The literaturereferenced time to maximum concentration for TAF is $\sim 0.5$ hours (Ruane et al., 2013) and would not have been adequately captured in the PK model had the absorption rate constant, $K_{\mathrm{a}}$, not been fixed. Since data for TAF were only available for three time points and the last time point had several values below the quantification limit, a TAF plasma model could not precisely be developed. A two-compartment model has been reported (Gaur et al., 2016), but was not supported by our data. Due to these limitations, a onecompartment model was used. The lack of data and/or compartmental misfit could explain the high IIV of TAF clearance and the inability to estimate volume IIV. However, the M3 method is the gold standard when modeling data sets
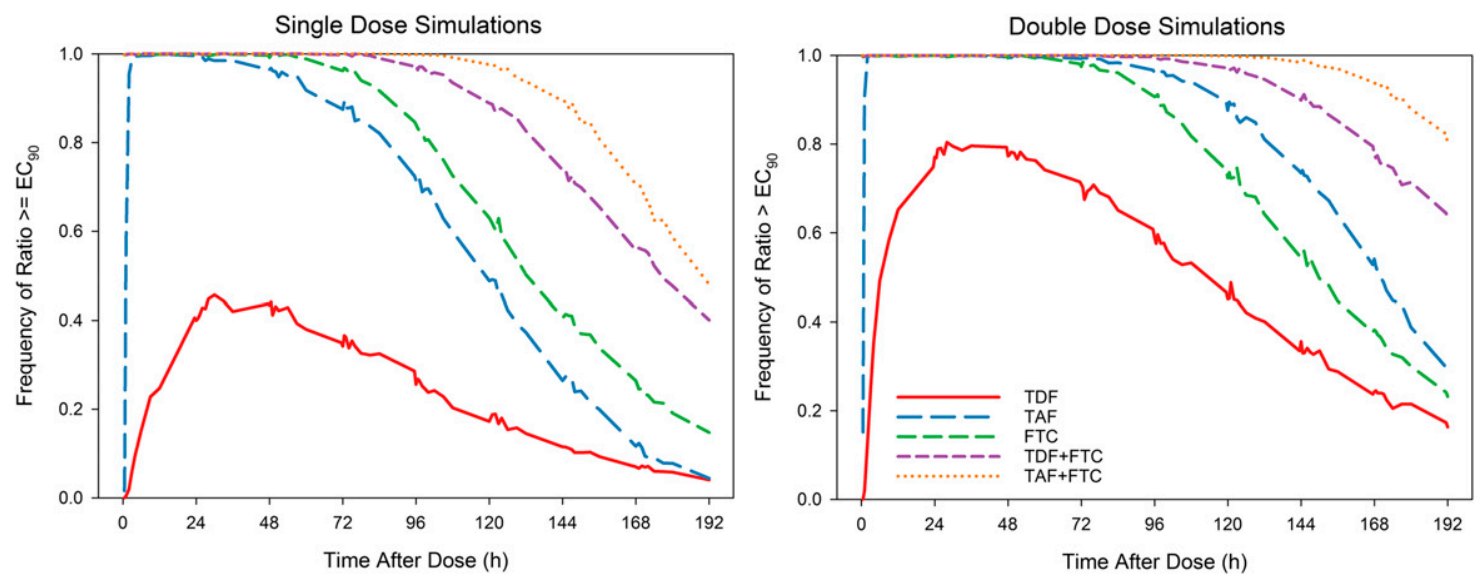

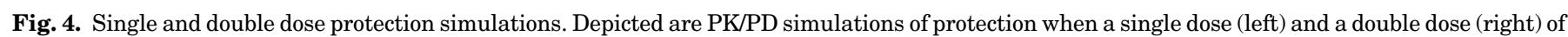
the indicated drug(s) is taken. Standard dose: $300 \mathrm{mg}$ TDF, $25 \mathrm{mg}$ TAF, $200 \mathrm{mg}$ FTC. 
with large proportions of values below the quantification limit (Beal, 2001), and was used to maximize the knowledge gained from these data. The model also assumes that the conversion of TAF to TFVdp and the subsequent elimination of TFVdp are linear across the 5-50 mg dose range, which may not be the case based on early steady-state data (Ruane et al., 2013). However, the single-dose data used in this analysis were linear (Cottrell et al., 2017). This raises an important consideration when using single-dose data for multidose simulations: elimination may not continue to be linear as concentrations increase. Unfortunately, the model was not able to account for this. However, if concentrations of TFVdp in PBMCs did increase due to nonlinear elimination, the model developed would underpredict efficacy rather than overpredict it, making this a conservative estimate of PrEP efficacy in a PWID population.

The paucity of phase III clinical data to evaluate the efficacy of PrEP among PWID limits the ability of clinicians to present their patients with scientifically informed options to prevent HIV infection. The Bangkok Tenofovir Study used a modifiedintent-to-treat analysis in which two participants were excluded because they were HIV positive at enrollment, and found a $48.9 \%$ reduction in HIV. Among participants adherent at least $71 \%$ of the time, without missing two or more consecutive doses, and who had detectable drug concentrations, there was a $73.5 \%$ reduction in HIV transmission in the TDF group (Choopanya et al., 2013). This corresponded to our analysis in which $71.2 \%$ and $82.5 \%$ of the population is expected to be protected with four to five doses of TDF per week. This is particularly striking because our analysis was based on the PK profiles of young, healthy women and not persons chronically ill from injection drug use. While acute kidney injury has been noted as a sequela of injection drug use (Wilson et al., 2017), normal renal function was assumed in this analysis. Other population models for TFV and FTC pharmacokinetics include a covariate effect of creatinine clearance on parent drug clearance. Such a function could be considered for this model; however, given that clinical studies evaluating TDF + FTC for PrEP exclude participants with reduced renal function-the Bangkok Tenofovir Study included-we did not implement this covariate in our model. Thus, any renal impairments associated with injection drug use in real-world patients would need to be taken into consideration, since the relationship between renal impairment and increasing TFV concentrations, and subsequent toxicity, is well-described (Hall et al., 2011). However, in individuals with creatinine clearance $>50 \mathrm{ml} / \mathrm{min}$, these medications require no dosage adjustment and have been used long-term with an acceptable adverse effect profile. The safety is such that the Food and Drug Administration recently approved TDF + FTC for long-term use in adolescents for PrEP (Office of Communication, 2018), an age cohort where the risk-benefit relationship was not always clear. In those with creatinine clearance $<50 \mathrm{ml} / \mathrm{min}$, continued use of TDF, particularly for PrEP, requires an individual weighing of HIV acquisition risk compared with risk of further renal impairment. TAF, on the other hand, has a more favorable safety profile compared with TDF, especially in regard to renal function (Ruane et al., 2013). Future work using the strategies highlighted in this modeling exercise in the PWID population will be needed to ensure our parameter estimates from healthy volunteers are comparable.
The length of PrEP dosing required to provide adequate protection is a concern. While we show that two doses per week of TDF + FTC or TAF + FTC would provide near 100\% protection, we did not specifically analyze the duration of time at which the ratio must exceed the efficacy target or the effect of multiple HIV exposures within a dosing interval. However, since a single dose would provide protection for 84 and 108 hours, we believe as long as the second dose is taken within that time frame protection should be maximal.

Finally, this analysis has been conducted with the assumption that PWID are only exposed to HIV via injection drug use and not via other concurrent routes. This likely does not apply to all PWID, and should be considered in the context of dosing strategies to protect mucosal surfaces for HIV infection (Cottrell et al., 2016). Women are particularly vulnerable and less than daily dosing has not been demonstrated to be effective for vaginal HIV exposure. Furthermore, less than daily dosing may be more challenging to adhere to (Bekker et al., 2018). A person's entire risk profile should be taken into account when considering PrEP.

In summary, to our knowledge, this is the first PK/PD model to evaluate TDF, TAF, and FTC for PrEP in a PWID population. We demonstrate data consistent with the Bangkok Tenofovir Study and provide characterization of the intracellular interactions necessary to protect cells from HIV infection. These data can be used to inform future clinical studies and potentially policy decisions in the absence of specific clinical investigations. This approach can also be extended to evaluate the efficacy of dosing scenarios for other compounds under investigation for PrEP. Because of the limitations highlighted, these data should not be used to make clinical decisions at this time, but should be used to design clinical studies to evaluate PrEP for PWID.

\section{Acknowledgments}

We thank the participants of the studies as well as the personnel of the clinical trials research center at the University of North Carolina. We also acknowledge Dr. Angela Kashuba for critically reviewing the data and manuscript.

\section{Authorship Contributions}

Participated in research design: Cottrell, Prince, Dumond. Conducted experiments: Garrett, Cottrell, Sykes, Schauer, White. Performed data analysis: Garrett, Chen, Maas, Cottrell.

Wrote or contributed to the writing of the manuscript: Garrett, Chen, Maas, Dumond.

\section{References}

Altice FL, Maru DSR, Bruce RD, Springer SA, and Friedland GH (2007) Superiority of directly administered antiretroviral therapy over self-administered therapy among HIV-infected drug users: a prospective, randomized, controlled trial. Clin Infect Dis 45:770-778.

Anderson PL, Kiser JJ, Gardner EM, Rower JE, Meditz A, and Grant RM (2011) Pharmacological considerations for tenofovir and emtricitabine to prevent HIV infection. J Antimicrob Chemother 66:240-250.

Baeten JM, Donnell D, Ndase P, Mugo NR, Campbell JD, Wangisi J, Tappero JW, Bukusi EA, Cohen CR, Katabira E, et al.; Partners PrEP Study Team (2012) Antiretroviral prophylaxis for HIV prevention in heterosexual men and women. $N$ Engl J Med 367:399-410.

Beal SL (2001) Ways to fit a PK model with some data below the quantification limit. $J$ Pharmacokinet Pharmacodyn 28:481-504.

Bekker LG, Roux S, Sebastien E, Yola N, Amico KR, Hughes JP, Marzinke MA, Hendrix CW, Anderson PL, Elharrar V, et al.; HPTN 067 (ADAPT) Study Team (2018) Daily and non-daily pre-exposure prophylaxis in African women (HPTN 067/ADAPT Cape Town trial): a randomised, open-label, phase 2 trial. Lancet HIV 5:e68-e78.

Berg KM, Litwin A, Li X, Heo M, and Arnsten JH (2011) Directly observed antiretroviral therapy improves adherence and viral load in drug users attending methadone maintenance clinics: a randomized controlled trial. Drug Alcohol Depend 113:192-199. 
Chen X, Seifert SM, Castillo-Mancilla JR, Bushman LR, Zheng JH, Kiser JJ, MaWhinney S, and Anderson PL (2016) Model linking plasma and intracellular tenofovir/emtricitabine with deoxynucleoside triphosphates. PLoS One 11: e0165505.

Choopanya K, Martin M, Suntharasamai P, Sangkum U, Mock PA, Leethochawalit M, Chiamwongpaet S, Kitisin P, Natrujirote P, Kittimunkong S, et al.; Bangkok Tenofovir Study Group (2013) Antiretroviral prophylaxis for HIV infection in injecting drug users in Bangkok, Thailand (the Bangkok Tenofovir study): a randomised, double-blind, placebo-controlled phase 3 trial. Lancet 381:2083-2090.

Cottrell ML, Garrett KL, Prince HMA, Sykes C, Schauer A, Emerson CW, Peery A, Rooney JF, McCallister S, Gay C, et al. (2017) Single-dose pharmacokinetics of tenofovir alafenamide and its active metabolite in the mucosal tissues. J Antimicrob Chemother 72:1731-1740.

Cottrell ML, Srinivas N, and Kashuba ADM (2015) Pharmacokinetics of antiretrovirals in mucosal tissue. Expert Opin Drug Metab Toxicol 11:893-905.

Cottrell ML, Yang KH, Prince HMA, Sykes C, White N, Malone S, Dellon ES, Madanick RD, Shaheen NJ, Hudgens MG, et al. (2016) A translational pharmacology approach to predicting outcomes of preexposure prophylaxis against HIV in men and women using tenofovir disoproxil fumarate with or without emtricitabine. $J$ Infect Dis 214:55-64.

Dickinson L, Yapa HM, Jackson A, Moyle G, Else L, Amara A, Khoo S, Back D, Karolia Z, Higgs C, et al. (2015) Plasma tenofovir, emtricitabine, and rilpivirine and intracellular tenofovir diphosphate and emtricitabine triphosphate pharmacokinetics following drug intake cessation. Antimicrob Agents Chemother 59: 6080-6086.

García-Lerma JG, Aung W, Cong ME, Zheng Q, Youngpairoj AS, Mitchell J, Holder A, Martin A, Kuklenyik S, Luo W, et al. (2011) Natural substrate concentrations can modulate the prophylactic efficacy of nucleotide HIV reverse transcriptase inhibitors. $J$ Virol 85:6610-6617.

Gaur AH, Kizito H, Prasitsueubsai W, Rakhmanina N, Rassool M, Chakraborty R, Batra J, Kosalaraksa P, Luesomboon W, Porter D, et al. (2016) Safety, efficacy, and pharmacokinetics of a single-tablet regimen containing elvitegravir, cobicistat emtricitabine, and tenofovir alafenamide in treatment-naive, HIV-infected adolescents: a single-arm, open-label trial. Lancet HIV 3:e561-e568.

Grant RM, Lama JR, Anderson PL, McMahan V, Liu AY, Vargas L, Goicochea P, Casapía M, Guanira-Carranza JV, Ramirez-Cardich ME, et al.; iPrEx Study Team (2010) Preexposure chemoprophylaxis for HIV prevention in men who have sex with men. $N$ Engl J Med 363:2587-2599.

Hall AM, Hendry BM, Nitsch D, and Connolly JO (2011) Tenofovir-associated kidney toxicity in HIV-infected patients: a review of the evidence. Am J Kidney Dis 57:773-780.

Keizer RJ, van Benten M, Beijnen JH, Schellens JHM, and Huitema ADR (2011) Piraña and PCluster: a modeling environment and cluster infrastructure for NONMEM. Comput Methods Programs Biomed 101:72-79.

Knox DC, Anderson PL, Harrigan PR, and Tan DHS (2017) Multidrug-resistant HIV-1 infection despite preexposure prophylaxis. N Engl J Med 376:501-502.
Lindbom L, Pihlgren P, and Jonsson EN (2005) PsN-Toolkit-a collection of computer intensive statistical methods for non-linear mixed effect modeling using NONMEM [published correction appears in Comput Methods Programs Biomed (2005) 80(3): 277]. Comput Methods Programs Biomed 79:241-257.

Molina JM, Capitant C, Spire B, Pialoux G, Cotte L, Charreau I, Tremblay C, Le Gall JM, Cua E, Pasquet A, et al.; ANRS IPERGAY Study Group (2015) On-demand preexposure prophylaxis in men at high risk for HIV-1 infection. $N$ Engl J Med 373:2237-2246.

Office of Communication (Press release, May 16, 2018) Item of Interest: FDA Approves PrEP therapy for adolescents at risk of HIV. National Institute of Child Health and Human Development, Rockville, MD.

Patterson KB, Prince HA, Kraft E, Jenkins AJ, Shaheen NJ, Rooney JF, Cohen MS and Kashuba ADM (2011) Penetration of tenofovir and emtricitabine in mucosal tissues: implications for prevention of HIV-1 transmission. Sci Transl Med 3: 112 re4.

R Core Team (2016) R: A Language and Environment for Statistical Computing, $\mathrm{R}$ Foundation for Statistical Computing, Vienna, Austria.

Rodriguez JF, Rodriguez JL, Santana J, García H, and Rosario O (2000) Simultaneous quantitation of intracellular zidovudine and lamivudine triphosphates in human immunodeficiency virus-infected individuals. Antimicrob Agents Chemother 44:3097-3100.

Ruane PJ, DeJesus E, Berger D, Markowitz M, Bredeek UF, Callebaut C, Zhong L, Ramanathan S, Rhee MS, Fordyce MW, et al. (2013) Antiviral activity, safety, and pharmacokinetics/pharmacodynamics of tenofovir alafenamide as 10-day monotherapy in HIV-1-positive adults. J Acquir Immune Defic Syndr 63:449-455.

US Public Health Service (2014) Preexposure prophylaxis for the prevention of HIV infection in the United States-2014, a clinical practice guideline. https://www.cdc. gov/hiv/pdf/guidelines/PrEPguidelines2014.pdf.

Wainberg MA, Moisi D, Oliveira M, Toni TD, and Brenner BG (2011) Transmission dynamics of the M184V drug resistance mutation in primary HIV infection. $J$ Antimicrob Chemother 66:2346-2349.

Wertheim JO, Oster AM, Johnson JA, Switzer WM, Saduvala N, Hernandez AL, Hall HI, and Heneine W (2017) Transmission fitness of drug-resistant HIV revealed in a surveillance system transmission network. Virus Evol 3:vex008.

Wilson MW, Bonnecaze AK, Dharod A, and Miller PJ (2017) Analysis of intensive care unit admission and sequelae in patients intravenously abusing extendedrelease oral oxymorphone. South Med $J$ 110:217-222.

Address correspondence to: Julie B. Dumond, Division of Pharmacotherapy and Experimental Therapeutics, UNC Eshelman School of Pharmacy, University of North Carolina at Chapel Hill, 1093 Genetic Medicine Building, CB 7361, 120 Mason Farm Road, Chapel Hill, NC 27599. E-mail: jdumond@ unc.edu 